\title{
Wavelet Channel Coding: An Algebraic Approach
}

\author{
João Fonseca Neto, Leocarlos B. S. Lima, and Francisco M. de Assis
}

\begin{abstract}
In this paper, the wavelet channel coding (WCC) is revisited in a more general analysis embracing flat real wavelet matrices derived from a Haar matrix and complex input symbols. WCC encoding and decoding are algebraically described and a probability distribution of wavelet symbols is formulated. Signal constellations for transmission of wavelet symbols are proposed and the constellation average energy is deduced from probability generating functions of the wavelet symbols. System performance over a flat Rayleigh channel is analyzed and compared with symbol-by-symbol detecting systems and diversity two space-time block coding (STBC) systems. Simulation results show that WCC presents better performance than ordinary symbol-by-symbol detecting systems, particularly at higher signal-to-noise ratios for higher spectral efficiencies, and STBC systems for spectral efficiency of $1 \mathrm{bit} / \mathrm{s} / \mathrm{Hz}$.
\end{abstract}

Index Terms-Communication systems, Rayleigh channels, wavelet transforms, adaptive systems, correlators, communication system performance.

\section{INTRODUCTION}

Wavelet theory has influenced several areas, such as: efficient approaches for representing functions in terms of a basis, compression systems concentrating most of the energy in a few coefficients, and channel coding methods [1]. However, literature is scarce on the application of wavelet matrices for communication channel coding, known as wavelet channel coding (WCC). It was originally proposed by Tzannes brothers in [2], coining their technique with this denomination (so we kept it).

Indeed, WCC is not properly a channel coding approach, since it does not have a distance profile between sequences of symbols and decoding is performed symbol-by-symbol. It yields an effect similar to an interleaving system and it does not

J. Fonseca Neto is with the Instituto Federal de Sergipe - IFS (Federal Institute of Sergipe), State of Sergipe, Brazil (e-mail: joao.fonseca@ee.ufcg.edu.br).

L. B. S. Lima and F. M. de Assis are with the Department of Electrical Engineering - DEE at Universidade Federal de Campina Grande - UFCG (Federal University of Campina Grande), State of Paraíba, Brazil, (e-mail: \{leocarlos, fmarcos\}@ dee.ufcg.edu.br).

Digital Object Identifier: 10.14209/jcis.2020.27 provide any coding gain over an additive white Gaussian noise channel.

Articles on the performance of systems that employ WCC can be found at [5], [6], [17], [20]. In general, WCC provides an increase in system performance at the cost of a large increase in the dimensionality of the output code. In order to counteract this drawback, some works propose constellations and increase spectral efficiency to wavelet symbols transmission, as in [8], [12], [13], [15].

WCC encoding is performed through a finite impulse response filter-like state machine composed by shift registers to store input symbols, coefficient taps from a wavelet coding matrix and adders. Information from each input symbol is spread over several wavelet symbols [1]. The consistent orthogonality between wavelet matrix rows, which remains even after overlapping and addition between them, makes possible to decode simply by using a bank of correlators formed from the rows of the matrix itself. This computational simplicity in decoding, with the same low complexity of the encoding process, and the ability to protect information from communication channel fading are the main advantages in employing WCC [2]. Furthermore, a coding rate change in WCC may be achieved by simple rearrangement of the coefficient taps and adders in the encoder, which suggests its use in adaptive systems to channel conditions. Alternative encoding and decoding approaches for WCC-based systems are explored in [9], [10], [16].

A WCC encoder maps a sequence of equiprobable input symbols to a sequence of not equiprobable wavelet symbols. The maximum number of wavelet symbols that carry part of the information from an input symbol is known as the constraint length of the wavelet code and is given by the product of the coding rate with the number of columns of the wavelet matrix [1]. An increase in input symbols cardinality or in constraint length has a strong impact on the cardinality of the wavelet symbol set and consequently on the cardinality of the transmission 
signal constellation [7]. In contrast, encoding and decoding complexities vary just linearly with the number of columns of the wavelet matrix.

WCC is presented and analyzed here in a more general approach, with WCC encoding and decoding algebraically described. The proposed system consider flat real wavelet matrices derived from a Haar matrix and complex input symbols. These input symbols are provided by a prior data mapper, which lets to increase the spectral efficiency, ordinarily restricted to $1 \mathrm{bit} / \mathrm{s} / \mathrm{Hz}^{1}$ [1]. A probability distribution of wavelet symbols is formulated. Signal constellations for transmission of wavelet symbols are proposed and the constellation average energy is deduced from probability generating functions of the wavelet symbols.

In order to infer about the employability of this technique, its performance was analyzed and compared with ordinary symbol-by-symbol detecting systems. In addition, WCC system performance was compared with space-time block coding (STBC) systems with two transmitting and one receiving antennas proposed by Siavash Alamouti in [21].

Section II briefly reviews the concepts on wavelet matrices. Section III presents the proposed WCC system, an algebraic description of WCC encoding and decoding processes, as well as the probability distribution of the generated wavelet symbols. Section IV proposes signal constellations for transmission of the WCC symbols. In addition, it calculates probability generating functions for the generated wavelet symbols and employs them to deduce the average energy of these constellations. Section V evaluates the performance of the proposed WCC system. At last, Section VI presents the conclusions for this work.

\section{WAVELET MATRIX}

A matrix $\mathbf{A}=\left[a_{k}^{s}\right]$ with $m \geq 2$ rows and $m g$ columns, such that

$$
\mathbf{A}=\left[\begin{array}{cccc}
a_{0}^{0} & a_{1}^{0} & \cdots & a_{m g-1}^{0} \\
a_{0}^{1} & a_{1}^{1} & \cdots & a_{m g-1}^{1} \\
\vdots & \vdots & \ddots & \vdots \\
a_{0}^{m-1} & a_{1}^{m-1} & \cdots & a_{m g-1}^{m-1}
\end{array}\right]
$$

\footnotetext{
${ }^{1}$ Excepting the approach proposed in [13], [14], where a constellation formed by pairs of consecutive integer wavelet symbols increased the spectral efficiency to $2 \mathrm{bits} / \mathrm{s} / \mathrm{Hz}$.
}

and $a_{k}^{s} \in \mathbb{R}$ or $\mathbb{C}$, is said to be a wavelet matrix or matrix of wavelet coefficients of rank $m$ and genus $g$ if the linear condition

$$
\sum_{k=0}^{m g-1} a_{k}^{s}=m \delta_{s, 0}, \quad 0 \leq s \leq m-1,
$$

and the quadratic condition

$$
\begin{aligned}
& \sum_{k=0}^{m g-1} a_{\left[k+m r^{\prime}\right]}^{s^{\prime}} \bar{a}_{[k+m r]}^{s}=m \delta_{s^{\prime}, s} \delta_{r^{\prime}, r}, \\
& \quad 0 \leq s^{\prime}, s \leq m-1 \quad \text { e } \quad 0 \leq r^{\prime}, r \leq g-1,
\end{aligned}
$$

are met, where $[k+m r]$ denotes the operation $k+$ $m r \bmod m g, \bar{a}$ is the complex conjugate of $a$ and $\delta_{x, y}$ is the Kronecker delta [1].

Equation (2) ensures that the sum of components of the first wavelet matrix row is equal to the rank $m$, whereas the sum of the components of each other row is zero. Equation (3) establishes that vectors represented by the $m$ wavelet matrix rows are orthogonal to each other, even when shifted by an arbitrary multiple of $m$. Equation (3) also indicates that each row in this matrix is orthogonal to itself when shifted by a multiple step of $m$.

A wavelet matrix is said to be flat when all elements have a same absolute value. If they are real numbers it is said to be a flat real wavelet matrix. If they are complex numbers it is said to be a flat complex wavelet matrix [1].

A flat real wavelet matrix with normalized elements satisfy the modified wavelet linear and quadratic conditions [1], [2]

$$
\sum_{k=0}^{m g-1} a_{k}^{s}=m \sqrt{g} \delta_{s, 0},
$$

and

$$
\sum_{k=0}^{m g-1} a_{\left[k+m r^{\prime}\right]}^{s^{\prime}} a_{[k+m r]}^{s}=m g \delta_{s^{\prime}, s} \delta_{r^{\prime}, r}
$$

We considered here just flat real normalized wavelet matrices derived from the Haar matrix

$$
\left[\begin{array}{cc}
1 & 1 \\
1 & -1
\end{array}\right]
$$

of rank $m=2$ and genus $g=1$. Rank may be increased to some power of 2 by tensor products as in $[1$, p. 50]. A genus $g$ may be raised to $4 g$ by an extension process described in [1, p. 54]. 


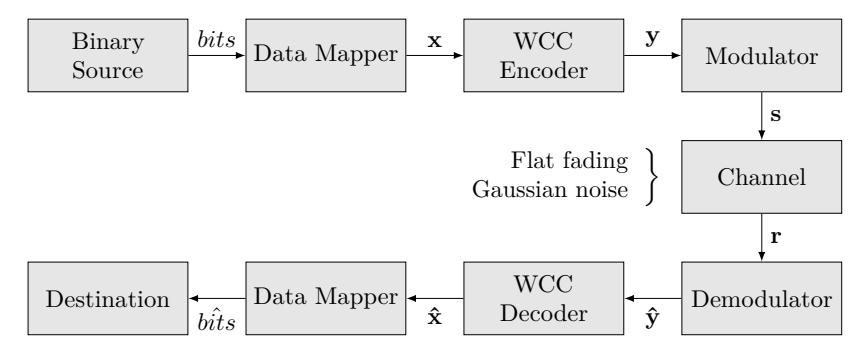

Fig. 1. Block diagram of a communication system employing WCC.

Example 1 (Wavelet coding matrix with rank $m=$ 2 and genus $g=4): \mathbf{A}_{2 \times 8}$ is given by

$$
\begin{aligned}
\mathbf{A} & =\left[\begin{array}{llllllll}
a_{0}^{0} & a_{1}^{0} & a_{2}^{0} & a_{3}^{0} & a_{4}^{0} & a_{5}^{0} & a_{6}^{0} & a_{7}^{0} \\
a_{0}^{1} & a_{1}^{1} & a_{2}^{1} & a_{3}^{1} & a_{4}^{1} & a_{5}^{1} & a_{6}^{1} & a_{7}^{1}
\end{array}\right] \\
& =\left[\begin{array}{cccccccc}
1 & 1 & 1 & -1 & 1 & 1 & -1 & 1 \\
1 & 1 & 1 & -1 & -1 & -1 & 1 & -1
\end{array}\right] .
\end{aligned}
$$

In simulations, the wavelet matrices $\mathbf{A}_{2 \times 8}(\mathrm{~m}=$ $2, g=4), \mathbf{A}_{2 \times 32}(m=2, g=16), \mathbf{A}_{2 \times 128}(m=$ $2, g=64), \mathbf{A}_{4 \times 16}(m=4, g=4), \mathbf{A}_{4 \times 64}(m=$ $4, g=16)$ and $\mathbf{A}_{4 \times 256}(m=4, g=64)$ were used.

\section{WAVELET CHANNEL CODING}

\section{A. System model}

We consider the communication system illustrated in Fig. 1.

As WCC works over any subfield of the complex field, it is natural to think about a more general representation of input information than in [2]. It is additionally interesting to recall the OFDM system, where input information is firstly formatted into complex symbols, so we followed the same idea.

In order to provide complex input symbols to the WCC Encoder (see Fig. 1), a Data Mapper was employed prior to the WCC Encoder to map $n_{b}$-bit sequences into $2^{n_{b}}$-ary complex symbol sequences $\mathbf{x}$, employing a Gray code, as illustrated in Table I. This mapping may not be the best one, but simulations suggest that simple rotations or expansions in the signal lattice (e.g., for $n_{b}=2,00 \rightarrow 1,01 \rightarrow j$, $11 \rightarrow-1$ and $10 \rightarrow-j$ ) does not alter results. Notice that this mapping from $\{0,1\}^{n_{b}}$ to $\mathbb{C}$ is common for classical systems (e.g., QAM), but in those cases points are mapping to the signal space directly. In the wavelet channel coding approach this step is mediated by a wavelet matrix.

In Fig. 1, input symbols in $\mathrm{x}$ are encoded forming wavelet symbols $\mathbf{y}$, according to some coding rate
TABLE I

DATA MAPPING PROCESS FROM $n_{b}$-BIT SEQUENCES INTO $2^{n_{b}}$-ARY COMPLEX SYMBOL SEQUENCES, WITH GRAY CODE.

\begin{tabular}{|c|c|c|c|c|c|}
\hline \multicolumn{2}{|c|}{$n_{b}=1$} & \multicolumn{2}{|c|}{$n_{b}=2$} & \multicolumn{2}{c|}{$n_{b}=4$} \\
\hline \hline 0 & -1 & 00 & $-1-j$ & 0000 & $-3-3 j$ \\
\hline 1 & 1 & 01 & $-1+j$ & 0001 & $-3-j$ \\
\hline & & 11 & $1+j$ & 0011 & $-3+j$ \\
\hline & & 10 & $1-j$ & 0010 & $-3+3 j$ \\
\hline & & & & 0110 & $-1+3 j$ \\
\hline & & & & 0111 & $-1+j$ \\
\hline & & & & 0101 & $-1-j$ \\
\hline & & & & 0100 & $-1-3 j$ \\
\hline & & & & 1100 & $1-3 j$ \\
\hline & & & & 1101 & $1-j$ \\
\hline & & & & 1111 & $1+j$ \\
\hline & & & & 1110 & $1+3 j$ \\
\hline & & & & 1010 & $3+3 j$ \\
\hline & & & & 1011 & $3+j$ \\
\hline & & & & 1001 & $3-j$ \\
\hline & & & & 1000 & $3-3 j$ \\
\hline
\end{tabular}

$R$. At last, wavelet symbols are modulated and transmitted through a flat Rayleigh fading channel. At the reception, a reverse process gives back an estimation of the source bits.

For simplicity, throughout this work, the scope of analysis was limited to $n_{b}=1$ and even values of $n_{b}$, as well as to $R=1$ and $R=1 / k$, considering $k$ an even integer. Odd values of $n_{b}$ and $k$ result in non-regular WCC encoders and asymmetrical constellations, without loss of generality of the presented analysis.

\section{B. Channel model}

In Fig. 1, symbols of a sequence $\mathbf{y}=$ $\left\{y_{0}, y_{1}, y_{2}, \ldots\right\}$ are transmitted serially. The channel is assumed to be a flat Rayleigh fading channel, where the path gain $\alpha_{n}$ at instant $n$ is modeled as samples of independent zero-mean complex Gaussian random variables with variance $1 / 2$ per axis.

At instant $n$, when a signal $s_{n}$ is transmitted, the received signal $r_{n}$ is given by

$$
r_{n}=\alpha_{n} s_{n}+\eta_{n}
$$

where noise samples $\eta_{n}$ are independent samples of a zero-mean complex Gaussian random variable with variance $E_{s} R /\left(2 n_{b} E_{b} / N_{0}\right)$ per dimension, where $E_{s}$ 
is the average energy of the transmission constellation and $E_{b} / N_{0}$ is the energy per bit to noise power spectral density ratio.

We assume that perfect channel state information is available at the receiver. So, the Demodulator module employs the decision metric

$$
\left|\frac{r_{n}}{\alpha_{n}}-s_{k}\right|^{2}
$$

over all possible transmitted signals $\left\{s_{k}\right\}$. The MAP (maximum a posteriori probability) criterion is adopted as decision rule.

\section{Algebraic WCC encoding}

Consider a binary source of i.i.d. equiprobable bits. Thus, the Data Mapper module yields a sequence of i.i.d. equiprobable symbols $\mathbf{x}=\left\{x_{0}, x_{1}, x_{2}, \ldots\right\}$. In order to describe algebraically the encoding process, $\mathbf{x}$ may be expressed as a formal series

$$
\mathbf{x}(D)=x_{0}+x_{1} D+x_{2} D^{2}+\cdots=\sum_{i=0}^{\infty} x_{i} D^{i},
$$

where $D$ represents a delay or shift operator, as in [3]. As $m$ symbols enter the encoder at a time, consider a serial to parallel operation prior to the encoding process and define the $m$ series

$$
\begin{aligned}
\mathbf{x}_{j}(D) & \triangleq x_{j}+x_{m+j} D+x_{2 m+j} D^{2}+\cdots \\
& =\sum_{i=0}^{\infty} x_{i m+j} D^{i}, \quad j \in\{0,1, \ldots, m-1\},
\end{aligned}
$$

which express the $m$ input symbols $\left\{x_{i m}, x_{i m+1}, \ldots\right.$, $\left.x_{(i+1) m-1}\right\}$ at an instant $i$. Remark that

$$
\mathbf{x}(D)=\sum_{j=0}^{m-1} \mathbf{x}_{j}\left(D^{m}\right) D^{j}
$$

In [2], the WCC encoding may be understood as a table operation, where $m$ input symbols multiply the $m$ rows of the wavelet coding matrix, a symbol for each line, and the $m^{2} g$ resulting values are stored in $m$ lines of the table. Then, $m / R$ wavelet symbols are obtained from the summations of the first $m / R$ columns of the table. Afterwards, the entire table is shifted by $m / R$ columns and the same operation is performed with the next $m$ input symbols to produce the next $m / R$ wavelet symbols. As a consequence of this process, $1 / g<R<1$. Tables II, III and IV illustrate this process for the cases of the wavelet coding matrix in (6) with coding rates $R=1,1 / 2$ and $1 / 4$, respectively.

According to this encoding process, in order to express algebraically the resulting wavelet symbols, consider the wavelet coding matrix $\mathbf{A}_{m \times m g}$ of rank $m$ and genus $g$ in (1) and define the matrix $m / R \times m$ of polynomials of degree $g R-1$

$$
\begin{aligned}
& \mathbf{A}(D) \triangleq \\
& {\left[\begin{array}{ccc}
\sum_{k=0}^{g R-1} a_{\frac{k m}{R}}^{0} D^{k} & \cdots & \sum_{k=0}^{g R-1} a_{\frac{k m}{R}}^{m-1} D^{k} \\
\sum_{k=0}^{g R-1} a_{\frac{k m}{R}+1}^{0} D^{k} & \cdots & \sum_{k=0}^{g R-1} a_{\frac{k m}{R}+1}^{m-1} D^{k} \\
\vdots & \ddots & \vdots \\
\sum_{k=0}^{g R-1} a_{\frac{k m}{R}+\frac{m}{R}-1}^{0} D^{k} & \cdots & \sum_{k=0}^{g R-1} a_{\frac{k m}{R}+\frac{m}{R}-1}^{m-1} D^{k}
\end{array}\right] .}
\end{aligned}
$$

So, a column vector of $m / R$ polynomials which own the $m / R$ wavelet symbols due to each $m$ input symbols may be given by

$$
\left[\begin{array}{c}
\mathbf{y}_{0}(D) \\
\mathbf{y}_{1}(D) \\
\vdots \\
\mathbf{y}_{m / R-1}(D)
\end{array}\right]=\mathbf{A}(D)\left[\begin{array}{c}
\mathbf{x}_{0}(D) \\
\mathbf{x}_{1}(D) \\
\vdots \\
\mathbf{x}_{m-1}(D)
\end{array}\right] .
$$

At last, considering a parallel to serial operation, the sequence of wavelet symbols $\mathbf{y}=\left\{y_{0}, y_{1}, y_{2}, \ldots\right\}$ may be obtained from (13) as

$$
\begin{aligned}
\mathbf{y}(D) & =y_{0}+y_{1} D+y_{2} D^{2}+\cdots=\sum_{i=0}^{\infty} y_{i} D^{i} \\
& =\sum_{j=0}^{m / R-1} \mathbf{y}_{j}\left(D^{m / R}\right) D^{j}
\end{aligned}
$$

Combining Equations (10), (11), (12), (13) and (14),

$$
\mathbf{y}(D)=\sum_{j=0}^{\frac{m}{R}-1} \sum_{l=0}^{m-1} \sum_{k=0}^{g R-1} \sum_{i=0}^{\infty} a_{\frac{k m}{R}+j}^{l} x_{i m+l} D^{\frac{(k+i) m}{R}+j} \text {. }
$$

Thus, the $n$-th wavelet symbol can be given by

$$
y_{n}=\sum_{\substack{n=\frac{(k+i) m}{R}+j \\ 0 \leq k<g R \\ i \geq 0 \\ 0 \leq j<\frac{m}{R}}} \sum_{l=0}^{m-1} a_{\frac{k m}{R}+j}^{l} x_{i m+l} .
$$

By inspection in (16), one observe that each wavelet symbol $y_{n}$ is derived from a linear combination of $m g R$ input symbols weighted by coefficients 
TABLE II

WCC ENCODING PROCESS FOR THE MATRIX IN (6), WITH RANK $m=2$, GENUS $g=4$ AND CODING RATE $R=1$.

\begin{tabular}{|c|c|c|c|c|c|c|c|c|}
\hline 0 & 1 & 2 & 3 & 4 & 5 & 6 & 7 & $\cdots$ \\
\hline \hline$x_{0} a_{0}^{0}$ & $x_{0} a_{1}^{0}$ & $x_{0} a_{2}^{0}$ & $x_{0} a_{3}^{0}$ & $x_{0} a_{4}^{0}$ & $x_{0} a_{5}^{0}$ & $x_{0} a_{6}^{0}$ & $x_{0} a_{7}^{0}$ & \\
\hline$x_{1} a_{0}^{1}$ & $x_{1} a_{1}^{1}$ & $x_{1} a_{2}^{1}$ & $x_{1} a_{3}^{1}$ & $x_{1} a_{4}^{1}$ & $x_{1} a_{5}^{1}$ & $x_{1} a_{6}^{1}$ & $x_{1} a_{7}^{1}$ & \\
\hline & & $x_{2} a_{0}^{0}$ & $x_{2} a_{1}^{0}$ & $x_{2} a_{2}^{0}$ & $x_{2} a_{3}^{0}$ & $x_{2} a_{4}^{0}$ & $x_{2} a_{5}^{0}$ & $\ldots$ \\
\hline & & $x_{3} a_{0}^{1}$ & $x_{3} a_{1}^{1}$ & $x_{3} a_{2}^{1}$ & $x_{3} a_{3}^{1}$ & $x_{3} a_{4}^{1}$ & $x_{3} a_{5}^{1}$ & $\ldots$ \\
\hline & & & & $x_{4} a_{0}^{0}$ & $x_{4} a_{1}^{0}$ & $x_{4} a_{2}^{0}$ & $x_{4} a_{3}^{0}$ & $\cdots$ \\
\hline & & & & $x_{5} a_{0}^{1}$ & $x_{5} a_{1}^{1}$ & $x_{5} a_{2}^{1}$ & $x_{5} a_{3}^{1}$ & $\cdots$ \\
\hline & & & & & & $x_{6} a_{0}^{0}$ & $x_{6} a_{1}^{0}$ & $\cdots$ \\
\hline \hline$y_{0}$ & $y_{1}$ & $y_{2}$ & $y_{3}$ & $y_{4}$ & $y_{5}$ & $y_{6}$ & $y_{7}$ & $\cdots$ \\
\hline
\end{tabular}

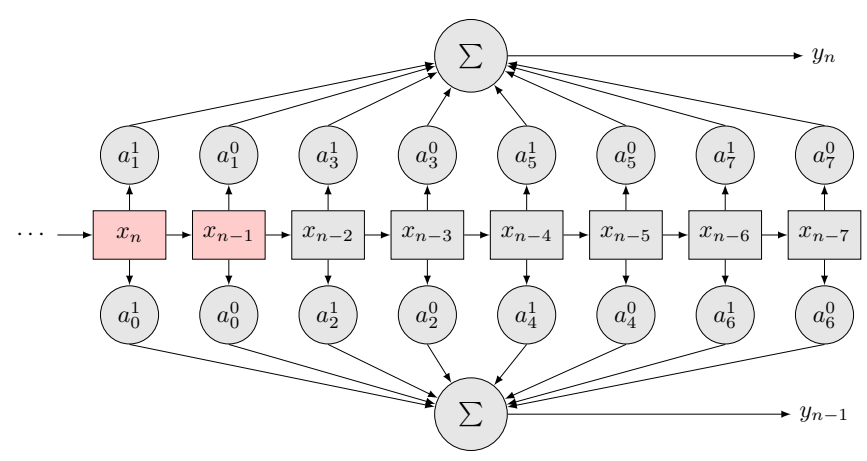

Fig. 2. WCC encoder diagram for the matrix in (6), with rank $m=2$, genus $g=4$ and coding rate $R=1$ (two input symbols in red and two output symbols at a time).

from the wavelet coding matrix. Each one of the $m^{2} g$ matrix coefficients are employed just once in the equations of the $\frac{m}{R}$ wavelet symbols derived from a block of $m$ input symbols. Also, real flat wavelet matrix coefficients, as considered here, have only values +1 or -1 (see (6) in Example 1). As a consequence, in the case of $n_{b}=1$, wavelet symbols are integer values $y_{n} \in\{-m g R,-m g R+$ $2, \ldots,-2,0,2, \ldots, m g R\}$.

Example 2 (WCC $2 \times 8$ with $R=1)$ : Consider the wavelet coding matrix in (6). Table II presents the encoding process and Fig. 2 shows the encoder diagram for a coding rate $R=1$.

The matrix of polynomials $\mathbf{A}(D)$ in (12) in this case is

$$
\mathbf{A}(D)=\left[\begin{array}{ll}
1+D+D^{2}-D^{3} & 1+D-D^{2}+D^{3} \\
1-D+D^{2}+D^{3} & 1-D-D^{2}-D^{3}
\end{array}\right]
$$

Thus, the wavelet symbols may be algebraically
TABLE III

WCC ENCODING PROCESS FOR THE MATRIX IN (6), WITH RANK $m=2$, GENUS $g=4$ AND CODING RATE $R=1 / 2$.

\begin{tabular}{|c|c|c|c|c|c|c|c|c|}
\hline 0 & 1 & 2 & 3 & 4 & 5 & 6 & 7 & $\cdots$ \\
\hline \hline$x_{0} a_{0}^{0}$ & $x_{0} a_{1}^{0}$ & $x_{0} a_{2}^{0}$ & $x_{0} a_{3}^{0}$ & $x_{0} a_{4}^{0}$ & $x_{0} a_{5}^{0}$ & $x_{0} a_{6}^{0}$ & $x_{0} a_{7}^{0}$ & \\
\hline$x_{1} a_{0}^{1}$ & $x_{1} a_{1}^{1}$ & $x_{1} a_{2}^{1}$ & $x_{1} a_{3}^{1}$ & $x_{1} a_{4}^{1}$ & $x_{1} a_{5}^{1}$ & $x_{1} a_{6}^{1}$ & $x_{1} a_{7}^{1}$ & \\
\hline & & & & $x_{2} a_{0}^{0}$ & $x_{2} a_{1}^{0}$ & $x_{2} a_{2}^{0}$ & $x_{2} a_{3}^{0}$ & $\cdots$ \\
\hline & & & & $x_{3} a_{0}^{1}$ & $x_{3} a_{1}^{1}$ & $x_{3} a_{2}^{1}$ & $x_{3} a_{3}^{1}$ & $\cdots$ \\
\hline \hline$y_{0}$ & $y_{1}$ & $y_{2}$ & $y_{3}$ & $y_{4}$ & $y_{5}$ & $y_{6}$ & $y_{7}$ & $\cdots$ \\
\hline
\end{tabular}

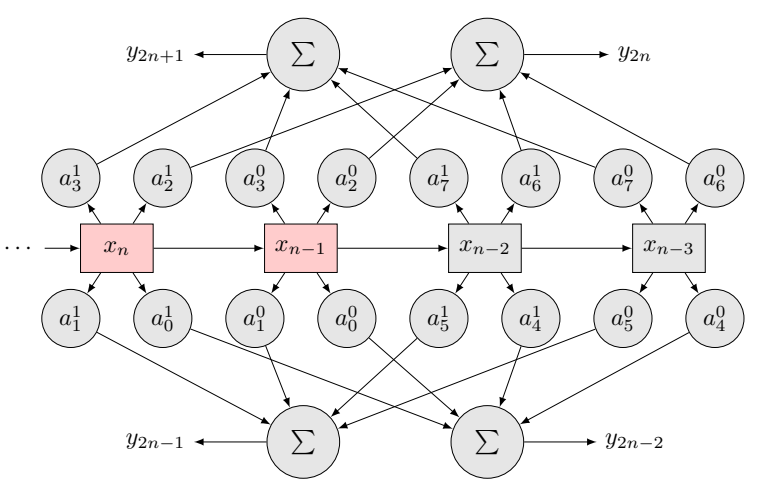

Fig. 3. WCC encoder diagram for the matrix in (6), with rank $m=2$, genus $g=4$ and coding rate $R=1 / 2$ (two input symbols in red and four output symbols at a time).

expressed as

$$
\begin{aligned}
& \mathbf{y}(D)=\mathbf{y}_{0}\left(D^{2}\right)+\mathbf{y}_{1}\left(D^{2}\right) D \\
& =\left[\begin{array}{ll}
1+D^{2}+D^{4}-D^{6} & 1+D^{2}-D^{4}+D^{6}
\end{array}\right] \text {. } \\
& {\left[\begin{array}{l}
x_{0}+x_{2} D^{2}+x_{4} D^{4}+\cdots \\
x_{1}+x_{3} D^{2}+x_{5} D^{4}+\cdots
\end{array}\right]+} \\
& {\left[1-D^{2}+D^{4}+D^{6} \quad 1-D^{2}-D^{4}-D^{6}\right] \text {. }} \\
& {\left[\begin{array}{l}
x_{0}+x_{2} D^{2}+x_{4} D^{4}+\cdots \\
x_{1}+x_{3} D^{2}+x_{5} D^{4}+\cdots
\end{array}\right] D} \\
& =x_{0}+x_{1}+\left(x_{0}+x_{1}\right) D+ \\
& \left(x_{0}+x_{1}+x_{2}+x_{3}\right) D^{2}+ \\
& \left(-x_{0}-x_{1}+x_{2}+x_{3}\right) D^{3}+ \\
& \left(x_{0}-x_{1}+x_{2}+x_{3}+x_{4}+x_{5}\right) D^{4}+ \\
& \left(x_{0}-x_{1}-x_{2}-x_{3}+x_{4}+x_{5}\right) D^{5}+\cdots
\end{aligned}
$$

Example 3 (WCC $2 \times 8$ with $R=1 / 2$ ): Consider the wavelet coding matrix in (6). Table III presents the encoding process and Fig. 3 shows the encoder diagram for a coding rate $R=1 / 2$.

The wavelet symbols may be algebraically ex- 
TABLE IV

WCC ENCODING PROCESS FOR THE MATRIX IN (6), WITH RANK $m=2$, GENUS $g=4$ AND CODING RATE $R=1 / 4$.

\begin{tabular}{|l|c|c|c|c|c|c|c|c|}
\hline$\cdots$ & 5 & 6 & 7 & 8 & 9 & 10 & 11 & $\cdots$ \\
\hline$\cdots$ & $x_{0} a_{5}^{0}$ & $x_{0} a_{6}^{0}$ & $x_{0} a_{7}^{0}$ & & & & & \\
\hline$\cdots$ & $x_{1} a_{5}^{1}$ & $x_{1} a_{6}^{1}$ & $x_{1} a_{7}^{1}$ & & & & & \\
\hline & & & & $x_{2} a_{0}^{0}$ & $x_{2} a_{1}^{0}$ & $x_{2} a_{2}^{0}$ & $x_{2} a_{3}^{0}$ & $\cdots$ \\
\hline & & & & $x_{3} a_{0}^{1}$ & $x_{3} a_{1}^{1}$ & $x_{3} a_{2}^{1}$ & $x_{3} a_{3}^{1}$ & $\cdots$ \\
\hline \hline$\cdots$ & $y_{5}$ & $y_{6}$ & $y_{7}$ & $y_{8}$ & $y_{9}$ & $y_{10}$ & $y_{11}$ & $\cdots$ \\
\hline
\end{tabular}

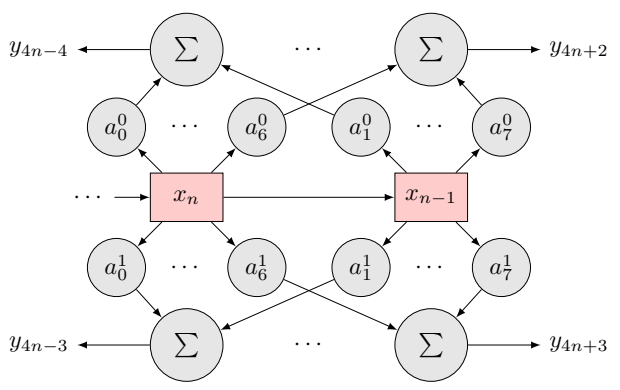

Fig. 4. WCC encoder diagram for the matrix in (6), with rank $m=2$, genus $g=4$ and coding rate $R=1 / 4$ (two input symbols in red and eight output symbols at a time).

pressed as

$$
\begin{aligned}
\mathbf{y}(D)= & \mathbf{y}_{0}\left(D^{4}\right)+\mathbf{y}_{1}\left(D^{4}\right) D+ \\
& \mathbf{y}_{2}\left(D^{4}\right) D^{2}+\mathbf{y}_{3}\left(D^{4}\right) D^{3} \\
= & x_{0}+x_{1}+\left(x_{0}+x_{1}\right) D+\left(x_{0}+x_{1}\right) D^{2}+ \\
& \left(-x_{0}-x_{1}\right) D^{3}+\left(x_{0}-x_{1}+x_{2}+x_{3}\right) D^{4}+ \\
& \left(x_{0}-x_{1}+x_{2}+x_{3}\right) D^{5}+ \\
& \left(-x_{0}+x_{1}+x_{2}+x_{3}\right) D^{6}+ \\
& \left(x_{0}-x_{1}-x_{2}-x_{3}\right) D^{7}+\cdots
\end{aligned}
$$

Example 4 (WCC $2 \times 8$ with $R=1 / 4$ ): Consider the wavelet coding matrix in (6). Table IV presents the encoding process and Fig. 4 shows the encoder diagram for a coding rate $R=1 / 4$.

The wavelet symbols may be algebraically expressed as

$$
\begin{aligned}
\mathbf{y}(D)= & x_{0}+x_{1}+\left(x_{0}+x_{1}\right) D+\left(x_{0}+x_{1}\right) D^{2}+ \\
& \left(-x_{0}-x_{1}\right) D^{3}+\left(x_{0}-x_{1}\right) D^{4}+ \\
& \left(x_{0}-x_{1}\right) D^{5}+\left(-x_{0}+x_{1}\right) D^{6}+ \\
& \left(x_{0}-x_{1}\right) D^{7}+\left(x_{2}+x_{3}\right) D^{8}+ \\
& \left(x_{2}+x_{3}\right) D^{9}+\left(x_{2}+x_{3}\right) D^{10}+\cdots
\end{aligned}
$$

An important remark about the WCC encoding process is that encoder diagrams for the same wavelet matrix and different coding rates just differ on the number of outputs (adders) and registers. Tap components stay the same, but in a different connection array. This feature suggests the employment of WCC where adaptability to channel conditions is desired.

\section{Probability distribution of wavelet symbols}

Considering a wavelet coding matrix of rank $m$ and genus $g$, coding rate $R=1$ and a data mapping for $n_{b}=1 \mathrm{bit} / \mathrm{symbol}$ (see Table I), there are

$$
N_{s}=m g R+1
$$

possible integer wavelet symbols

$y_{n} \in\{-m g R,-m g R+2, \ldots,-2,0,2, \ldots, m g R\}$.

If $n_{b}>1$, formatted input symbols are complex, consequently wavelet symbols are as well. In the data mapping proposed (see Table I), even values of $n_{b}>1$ are just considered, although odd values may be employed. In this case,

$$
\begin{aligned}
\operatorname{Re}\left\{y_{n}\right\}, & \operatorname{Im}\left\{y_{n}\right\} \in\left\{-m g R\left(2^{n_{b} / 2}-1\right),\right. \\
& -m g R\left(2^{n_{b} / 2}-1\right)+2, \ldots, \\
& \left.-2,0,2, \ldots, m g R\left(2^{n_{b} / 2}-1\right)\right\},
\end{aligned}
$$

so there are

$$
N_{s}=\left[m g R\left(2^{n_{b} / 2}-1\right)+1\right]^{2}
$$

possible complex wavelet symbols.

If input bits are i.i.d., formatted input symbols will also be i.i.d. Since wavelet coding is linear, wavelet symbols in (18) and (19) will be independent, but not equally distributed. In this case, when $n_{b}=1$, the wavelet symbols $\left\{y_{n}\right\}$ in (18) present a binomial probability distribution [2] given by

$$
\mathrm{P}\left[y_{n}=2 k-m g R\right]=\left(\begin{array}{c}
m g R \\
k
\end{array}\right) \frac{1}{2^{m g R}},
$$

where $0 \leq k \leq m g R$. For $n_{b}>1$, real and imaginary parts of the wavelet symbols in (19) are multinomially distributed (see subsection IV-B on the methodology employed to derive wavelet symbol probabilities from a probability generation function). 
Since $\operatorname{Re}\left\{y_{n}\right\}$ and $\operatorname{Im}\left\{y_{n}\right\}$ are independent of each other, then

$$
\begin{aligned}
& \mathrm{P}\left\{y_{n}=2 k-m g R\left(2^{n_{b} / 2}-1\right)+\right. \\
& \left.j\left[2 l-m g R\left(2^{n_{b} / 2}-1\right)\right]\right\}= \\
& \frac{1}{2^{m g R n_{b}}}\left[\sum_{k_{1}+\cdots+k_{2^{n_{b}} / 2}=m g R}\left(\begin{array}{c}
m g R \\
k_{1}, \ldots, k_{2^{n_{b} / 2}}
\end{array}\right)\right] \\
& {\left[\sum_{l_{1}+\cdots+l_{2^{n_{b}} / 2}=m g R}\left(\begin{array}{c}
m g R \\
l_{1}, \ldots, l_{2^{n_{b} / 2}}
\end{array}\right)\right],}
\end{aligned}
$$

where $j=\sqrt{-1}$ is the imaginary unit, $0 \leq k \leq$ $m g R\left(2^{n_{b} / 2}-1\right), 0 \leq l \leq m g R\left(2^{n_{b} / 2}-1\right)$ and the integer indexes $k_{1}, \ldots, k_{2^{n_{b} / 2}}$ and $l_{1}, \ldots, l_{2^{n_{b} / 2}}$ represent the yielded $\operatorname{Re}\left\{y_{n}\right\}$ and $\operatorname{Im}\left\{y_{n}\right\}$, respectively. Each index in $k_{1}, \ldots, k_{2^{n_{b} / 2}}$ (similarly in $l_{1}, \ldots, l_{2^{n_{b}} / 2}$ ) is associated to one of the $2^{\frac{n_{b}}{2}}$ formatted input symbols and means how many times the respective input symbol appears in the coder's register to produce $y_{n}$. As the coder has length $m g R$, so $k_{1}+\cdots+k_{2^{n_{b} / 2}}=m g R$.

\section{E. Correlation WCC decoding}

Input symbols $\mathrm{x}$ may be estimated by correlation of the last $m g$ received wavelet symbols $\hat{\mathbf{y}}$ with the rows of the wavelet coding matrix [2]. Each new $m / R$ received wavelet symbols $\hat{\mathbf{y}}$ produce $m$ symbols $\hat{\mathbf{x}}$ at the output of the WCC Decoder module.

Denote by $z_{r}^{s}$ the correlation between the $s$-th line of the wavelet matrix and the last $m g$ received wavelet symbols $\hat{\mathbf{y}}$ at instant $r$, such that

$$
z_{r}^{s}=\sum_{t=0}^{m g-1} a_{m g-1-t}^{s} y_{r-t} .
$$

From (16),

$$
z_{r}^{s}=\sum_{t=0}^{m g-1} \sum_{\substack{r-t=\frac{(k+i) m}{R} \\ 0 \leq k<g R \\ i \geq 0 \\ i \geq 0 \\ 0 \leq j<\frac{m}{R}}} \sum_{l=0}^{m-1} a_{m g-1-t}^{s} a_{\frac{k m}{R}+j}^{l} x_{i m+l} .
$$

Considering condition (5), which guarantees that wavelet coding matrix rows are orthogonal between them even at shifts of $m$ columns, then all terms in

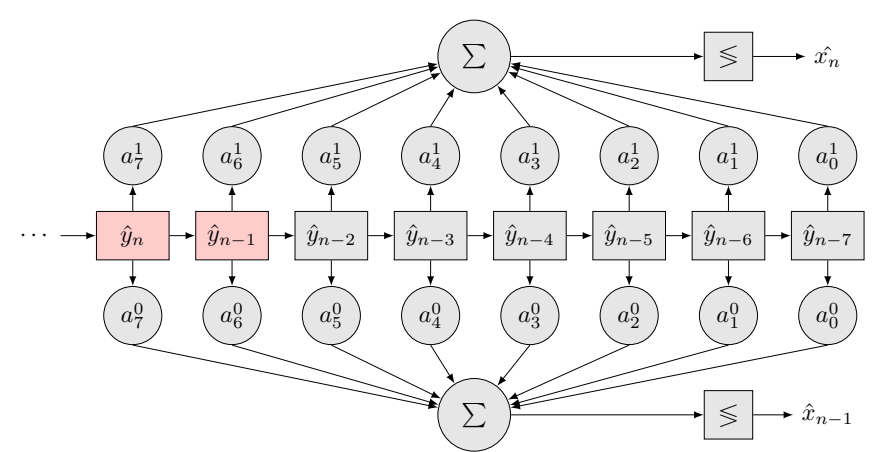

Fig. 5. Correlation WCC decoder diagram for the matrix in (6), with rank $m=2$, genus $g=4$ and coding rate $R=1$ (two input symbols in red and two output symbols at a time).

(23) cancel, except when $l=s$ and $(m g-1)-t=$ $\frac{k m}{R}+j$. Thus,

$$
\begin{aligned}
z_{r}^{s} & =x_{(r-m g+1) R+s} \sum_{u=0}^{m g-1} a_{u}^{s} a_{u}^{s} \\
& =m g x_{(r-m g+1) R+s} .
\end{aligned}
$$

Detailed descriptions of this decoding process for $R=1$ and $n_{b}=1$ can be found in [2], [7], [8], [17]-[20].

Example 5 (WCC $2 \times 8$ ): Consider the wavelet coding matrix in (6). Fig. 5 shows the correlation decoder diagram for a coding rate $R=1$. In fact, this decoder operates for any $R$, when it receives $2 / R$ input symbols and yields two output symbols at a time.

In Fig. 5, blocks $\lessgtr$ implement a decision logic over values of $z_{r}^{s}$ in (24).

\section{CONSTEllations For WCC Systems}

System performance depends directly on the modulation scheme. This Section presents signal constellations for transmitting wavelet symbols produced by the proposed system in Fig. 1. In the next Section, its performance is evaluated over a flat Rayleigh fading channel.

The proposed WCC system requires specially designed constellations since generated wavelet symbols are not equiprobable and the alphabet cardinality grows with the wavelet coding matrix order. The construction of special constellations was explored in [7], [8], [13], [14].

\section{A. Constellation model}

As introduced in Section I, an increase in the input symbols cardinality and in the code constraint 
length have a strong impact on the cardinality of the wavelet symbols set and consequently on the cardinality of the transmission signal constellation.

The WCC entropy rate is 1 bit/symbol when the coding rate is unitary and the spectral efficiency is limited to $1 \mathrm{bit} / \mathrm{s} / \mathrm{Hz}$ [1]. In order to improve this efficiency, a method to increase it to $2 \mathrm{bits} / \mathrm{s} / \mathrm{Hz}$ by constructing a signal constellation from consecutive pairs of integer wavelet symbols was proposed in [13]. These symbol pairs were directly associated to points in a signal space diagram forming a constellation for transmission. One observed that greater probability constellation points corresponded to lower energy signals. In [15], an optimizing effort suggested that these constellations were optimal. The method proposed in [13] could be generalized to blocks of length greater than two to achieve an arbitrary spectral efficiency, however the block-tosignal mapping would not be as simple as in the case of symbols pairs.

The data mapping proposed in Fig. 1 to generalize the WCC encoding and decoding algebraic formulations results in an alternative way to improve the spectral efficiency when $n_{b}>1$ (see Table I). The Modulator module directly associates a complex wavelet symbol $a+j b$ to a constellation point $(a, b)$. This method allows to achieve a spectral efficiency

$$
\eta=n_{b} R \quad(\text { bits } / \mathrm{s} / \mathrm{Hz})
$$

for a coding rate $R$. However, This approach entails an exponential growing of the constellation cardinality, since the number of possible wavelet symbols in (20) rises with $2^{n_{b}} R^{2}$ when $n_{b}>1$.

\section{B. Probability generating function of WCC symbols}

In [13], a probability generating function (PGF) for a wavelet symbol sequence was formulated. This alternative PGF permitted to predict integer symbol pairs generated by a WCC encoder and provided the joint probability distribution of these pairs. Following this methodology, a PGF that identifies the sequence of complex wavelet symbols generated in the system proposed here and their probability distribution are derived. From this PGF, one can also derive the average energy of the constellation.

Firstly, consider a binary source generating i.i.d. equiprobable bits. As a consequence, the Data Mapper module yields i.i.d. equiprobable input symbols $\mathbf{x}$ to the WCC Encoder (see Fig. 1). So, one can write the PGF of the $n$-th input symbol $x_{n}$ as

$$
\begin{aligned}
G_{x_{n}}(z) & =\mathrm{E}\left[z^{x_{n}}\right]=\sum_{i} z^{\alpha_{i}} P\left(x_{n}=\alpha_{i}\right) \\
& =\frac{1}{2^{n_{b}}} \sum_{i=0}^{2^{n_{b}-1}} z^{\alpha_{i}},
\end{aligned}
$$

where $\left\{\alpha_{i}\right\}$ is the set of all possible input symbols.

Example 6 (PGF of WCC Encoder input symbols from Table I): Consider the symbols generated by the Data Mapper according to the Table I. From (26), if $n_{b}=1$,

$$
G_{x_{n}}(z)=\frac{1}{2}\left(z^{1}+z^{-1}\right) .
$$

If $n_{b}=2$,

$$
G_{x_{n}}(z)=\frac{1}{4}\left[\left(z^{1+j}+z^{-1-j}\right)+\left(z^{1-j}+z^{-1+j}\right)\right] .
$$

If $n_{b}=4$,

$$
\begin{gathered}
G_{x_{n}}(z)=\frac{1}{16}\left[\left(z^{3+3 j}+z^{-3-3 j}\right)+\left(z^{3+j}+\right.\right. \\
\left.z^{-3-j}\right)+\left(z^{3-j}+z^{-3+j}\right)+\left(z^{3-3 j}+z^{-3+3 j}\right)+ \\
\left(z^{1+3 j}+z^{-1-3 j}\right)+\left(z^{1+j}+z^{-1-j}\right)+ \\
\left.\left(z^{1-j}+z^{-1+j}\right)+\left(z^{1-3 j}+z^{-1+3 j}\right)\right] .
\end{gathered}
$$

A common PGF of a sequence of $n$ wavelet symbols $\left\{y_{k+1}, \ldots, y_{k+n}\right\}$ is a power series representation of the joint probability distribution $\mathrm{P}\left(y_{k+1}, \ldots, y_{k+n}\right)$ of this sequence, given by

$$
\begin{aligned}
& G_{y_{k+1}, \ldots, y_{k+n}}\left(z_{1}, \ldots, z_{n}\right)=\mathrm{E}\left(z_{1}^{y_{k+1}} \cdots z_{n}^{y_{k+n}}\right) \\
& =\sum_{y_{k+1}, \ldots, y_{k+n}} \mathrm{P}\left(y_{k+1}, \ldots, y_{k+n}\right) z_{1}^{y_{k+1}} \cdots z_{n}^{y_{k+n}} .
\end{aligned}
$$

This is equivalent to the definition of a characteristic function of a random vector, as in [22, p. 255-256]. So, an ordinary PGF of a sequence of $n$ wavelet symbols can be viewed or interpreted as a mapping

$$
\mathbb{Z}^{n} \longrightarrow \mathbb{C}^{n}
$$

Consider now a sequence of wavelet symbols generated by the system expressed as a polynomial $\mathbf{y}(D)$ as in (15). One may consider, as in [13], an alternative definition of a PGF of a sequence of wavelet symbols as

$$
G_{\mathbf{y}(D)}(z)=\mathrm{E}\left(z^{\mathbf{y}(D)}\right),
$$


which employs a single complex variable $z$. In this last case, the PGF consists of a mapping

$$
\mathbb{Z}^{n} \longrightarrow \mathbb{Z}[D]
$$

where $\mathbb{Z}[D]$ is a ring of polynomials in $D$.

In the PGF in (26), as in the PGF of wavelet symbols deduced below, exponents of $z$ indicate the generated wavelet symbols and constants multiplying terms in $z$ inform the probability of each corresponding symbol. In (26), all symbols are equiprobable, but resultant wavelet symbols shall not be equiprobable.

If $\mathbf{y}(D)$ is the sequence of wavelet symbols generated by a WCC Encoder, from (15),

$$
\begin{aligned}
G_{\mathbf{y}(D)}(z) & =\mathrm{E}\left[z^{\mathbf{y}(D)}\right] \\
& =\mathrm{E}\left[z^{\sum_{i, j, k, l} a_{\frac{k m}{R}+j}^{l} x_{i m+l} D^{\frac{(k+i) m}{R}+j}}\right] .
\end{aligned}
$$

As input symbols are independent,

$$
\begin{aligned}
G_{\mathbf{y}(D)}(z) & =\prod_{i, l} \mathrm{E}\left[z^{\left.x_{i m+l} \sum_{j, k} a_{\frac{k m}{R}+j}^{l} D^{\frac{(k+i) m}{R}+j}\right]}\right. \\
& =\prod_{i, l} G_{x_{i m+l}}\left(z^{\sum_{j, k} a_{\frac{k m}{R}+j}^{l} D^{\frac{(k+i) m}{R}+j}}\right) .
\end{aligned}
$$

As input symbols are equiprobable and each wavelet symbol is a function of $m g R$ input symbols weighted by wavelet coding matrix coefficients (values +1 and -1 ), one can find for the $n$-th wavelet symbol in the generated sequence that

$$
G_{y_{n}}(z)=\frac{1}{2^{m g R n_{b}}}\left(\sum_{i=0}^{2^{n_{b}-1}} z^{\alpha_{i}}\right)^{m g R},
$$

where $\left\{\alpha_{i}\right\}$ is the set of all possible input symbols. Applying the multinomial theorem ${ }^{2}$ to solve the power of sum in (32),

$$
\begin{aligned}
G_{y_{n}}(z)=\frac{1}{2^{m g R n_{b}}} \sum_{k_{0}+\cdots+k_{2^{n} b-1}=m g R} \\
\quad\left(\begin{array}{c}
m g R \\
k_{0}, \ldots, k_{2^{n} b-1}
\end{array}\right) z^{\sum_{i=0}^{2^{n_{b}-1}} k_{i} \alpha_{i}} .
\end{aligned}
$$

${ }^{2}$ By the well-known multinomial theorem (generalization of the binomial theorem),

$$
\left(\sum_{i=1}^{m} x_{i}\right)^{n}=\sum_{k_{1}+k_{2}+\cdots+k_{m}=n}\left(\begin{array}{c}
n \\
k_{1}, k_{2}, \ldots, k_{m}
\end{array}\right) \prod_{i=1}^{m} x_{i}^{k_{i}},
$$

where $\left(\begin{array}{c}n \\ k_{1}, k_{2}, \ldots, k_{m}\end{array}\right)=\frac{n !}{k_{1} ! k_{2} ! \ldots k_{m} !}$ is the so-called multinomial coefficient.
In (33), as in (26), exponents of $z$ indicate generated wavelet symbols and constants multiplying each term inform the probability of the corresponding symbol. For $n_{b}>1$, wavelet symbols are complex and (33) may be changed to treat separately real and imaginary parts of the symbols, as in subsection III-D. There, (22) was derived to provide wavelet symbol probabilities with lower complexity.

Example 7 (PGF of wavelet symbols for a coding matrix $\mathbf{A}_{2 \times 8}$ and $\left.R=1 / 2\right)$ : Consider the wavelet coding matrix in (6) and a coding rate $R=1 / 2$.

Considering equally distributed input symbols, from (32), for $n_{b}=1$ in Table I,

$$
\begin{aligned}
G_{y_{n-3}}(z) & =G_{y_{n-2}}(z)=G_{y_{n-1}}(z)=G_{y_{n}}(z) \\
& =\frac{1}{2^{4}}\left(z^{1}+z^{-1}\right)^{4} .
\end{aligned}
$$

If $n_{b}=2$,

$$
\begin{aligned}
& \begin{array}{l}
G_{y_{n-3}}(z)=G_{y_{n-2}}(z)=G_{y_{n-1}}(z)=G_{y_{n}}(z) \\
=\frac{1}{2^{8}}\left[\left(z^{1+j}+z^{-1-j}\right)+\left(z^{1-j}+z^{-1+j}\right)\right]^{4} . \\
\text { If } n_{b}=4 \\
G_{y_{n-3}}(z)=G_{y_{n-2}}(z)=G_{y_{n-1}}(z)=G_{y_{n}}(z)= \\
\frac{1}{2^{16}}\left[\left(z^{3+3 j}+z^{-3-3 j}\right)+\left(z^{3+j}+z^{-3-j}\right)+\right. \\
\qquad\left(z^{3-j}+z^{-3+j}\right)+\left(z^{3-3 j}+z^{-3+3 j}\right)+ \\
\quad\left(z^{1+3 j}+z^{-1-3 j}\right)+\left(z^{1+j}+z^{-1-j}\right)+ \\
\left.\quad\left(z^{1-j}+z^{-1+j}\right)+\left(z^{1-3 j}+z^{-1+3 j}\right)\right]^{4} .
\end{array}
\end{aligned}
$$

From (33), for $n_{b}=2$,

$$
\begin{gathered}
G_{y_{n-3}}(z)=G_{y_{n-2}}(z)=G_{y_{n-1}}(z)=G_{y_{n}}(z)= \\
\frac{1}{256}\left(z^{-4-4 j}+z^{-4+4 j}+z^{4-4 j}+z^{4+4 j}\right)+ \\
\frac{1}{64}\left(z^{-4-2 j}+z^{-4+2 j}+z^{4-2 j}+z^{4+2 j}+z^{-2-4 j}+\right. \\
\left.z^{-2+4 j}+z^{2-4 j}+z^{2+4 j}\right)+\frac{3}{128}\left(z^{4}+z^{-4}+\right. \\
\left.z^{4 j}+z^{-4 j}\right)+\frac{1}{16}\left(z^{-2-2 j}+z^{-2+2 j}+z^{2-2 j}+\right. \\
\left.z^{2+2 j}\right)+\frac{3}{32}\left(z^{2}+z^{-2}+z^{2 j}+z^{-2 j}\right)+\frac{9}{64} z^{0} .
\end{gathered}
$$

Fig. 6 illustrates the resulting constellations and corresponding probability distribution curves for the above cases. 


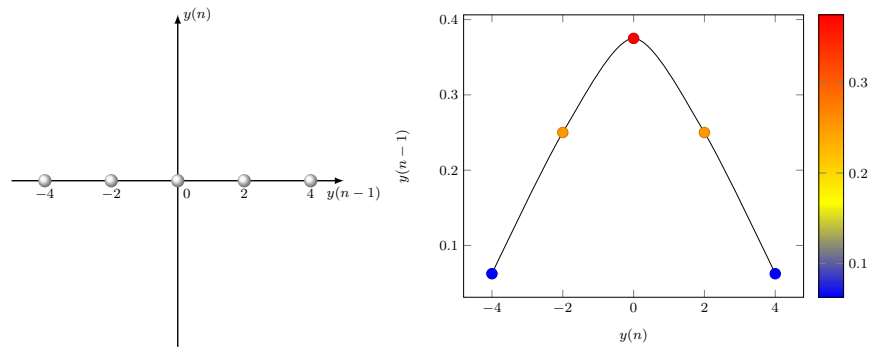

(a) For $n_{b}=1$, with 5 points and average energy $E_{s}=4$.
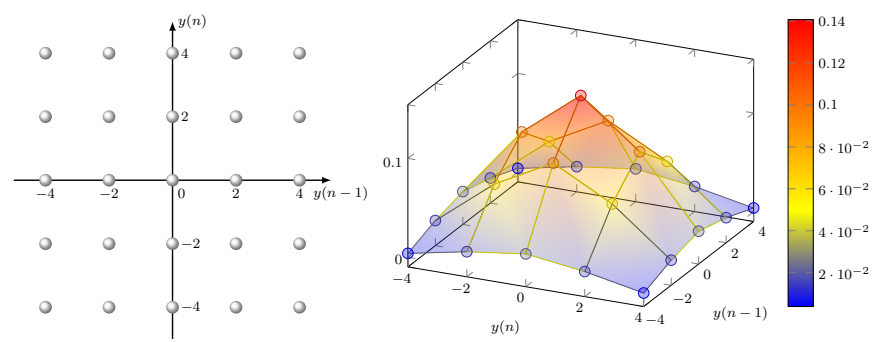

(b) For $n_{b}=2$, with 25 points and average energy $E_{s}=8$.
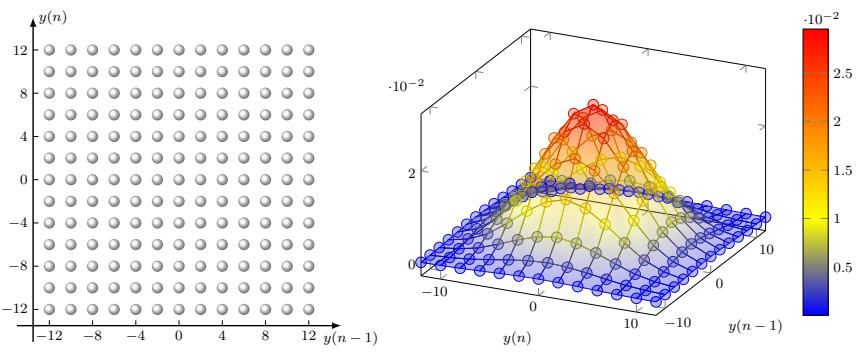

(c) For $n_{b}=4$, with 169 points and average energy $E_{s}=24$.

Fig. 6. Signal constellations and probability distribution curves for a WCC system with the coding matrix $\mathbf{A}_{2 \times 8}$ in (6) and $R=1 / 2$.

\section{Constellation average energy}

One can employ the wavelet symbols PGF to determine the average energy $E_{s}$ of the resulting constellation. The mean value of wavelet symbols, $\mathrm{E}\left[y_{n}\right]$, can be obtained evaluating the first derivative of the PGF in $z=1$ and $\mathrm{E}\left[y_{n}^{2}\right]-\mathrm{E}\left[y_{n}\right]$ can be calculated evaluating the second derivative of the PGF in $z=1$ [22]. One can remark that $E_{s}=\mathrm{E}\left[y_{n}^{2}\right]$.

Initially, consider the PGF in (32) when $n_{b}=1$, case of integer wavelet symbols. Its first derivative is

$$
G_{y}^{\prime}(z)=\frac{m g R}{2^{m g R}}\left(1-z^{-2}\right)\left(z+z^{-1}\right)^{m g R-1} .
$$

So,

$$
\mathrm{E}\left[y_{n}\right]=\left.G_{y}^{\prime}(z)\right|_{z=1}=0
$$

Thus, when $n_{b}=1$, the second derivative of the PGF in $z=1$ directly yields the average energy of the constellation.
The second derivative of (32) is

$$
\begin{aligned}
& G_{y}^{\prime \prime}(z)=\frac{m g R}{2^{m g R}}\left[2 z^{-3}\left(z+z^{-1}\right)^{m g R-1}+\right. \\
& \left.\left(1-z^{-2}\right)^{2}(m g R-1)\left(z+z^{-1}\right)^{m g R-2}\right] .
\end{aligned}
$$

So, the constellation average energy when $n_{b}=1$ is

$$
\begin{array}{r}
E_{s}=\left.G_{y}^{\prime \prime}(z)\right|_{z=1}=\frac{m g R}{2^{m g R}}\left[2 \cdot 1 \cdot 2^{m g R-1}+\right. \\
\left.0^{2} \cdot(m g R-1) 2^{m g R-2}\right]=m g R
\end{array}
$$

When $n_{b}>1$, wavelet symbols are complex. Consider now a complex wavelet symbol $y_{n}=u_{n}+j v_{n}$, where $u_{n}$ and $v_{n}$ are the real and imaginary parts of $y_{n}$. Observe that $u_{n}$ and $v_{n}$ are independent because $u_{n}$ is due to the real part of the input symbols and $v_{n}$ is due to their imaginary part. In addition, the real and imaginary parts of the input symbols may be associated to half the input bits in data mapping $\left(n_{b} / 2\right)$. So, $u_{n}$ and $v_{n}$ behave as $y_{n}$ when $n_{b}=1$ and $u_{n}, v_{n} \in\left\{-m g R 2^{n_{b} / 2},-m g R 2^{n_{b} / 2}+\right.$ $\left.2, \ldots, m g R 2^{n_{b} / 2}\right\}$. Then, the PGFs of $u_{n}$ and $v_{n}$ can be directly derived from (32) as

$$
\begin{aligned}
G_{u_{n}}(z)=G_{v_{n}}(z) & =\frac{1}{2^{m g R n_{b} / 2}} \\
& \left(\sum_{i=0}^{2^{n_{b} / 2}-1} z^{2 i-2^{n_{b} / 2}+1}\right)^{m g R} .
\end{aligned}
$$

In order to evaluate the mean of $u_{n}$ and $v_{n}$, as in the case of $n_{b}=1$, the first derivative of (38) is evaluated in $z=1$. So,

$$
\begin{aligned}
G_{u_{n}}^{\prime}(z)= & G_{v_{n}}^{\prime}(z)=\frac{1}{2^{m g R n_{b}}} m g R \\
& \left(\sum_{i=0}^{2^{n_{b}}-1} z^{2 i-2^{n_{b} / 2}+1}\right)^{m g R-1} \\
& \sum_{i=0}^{2^{n_{b}-1}}\left(2 i-2^{n_{b} / 2}+1\right) z^{2 i-2^{n_{b} / 2}}
\end{aligned}
$$

and

$$
\begin{array}{r}
\mathrm{E}\left[y_{n}\right]=\left.G_{y}^{\prime}(z)\right|_{z=1}=\frac{m g R}{2^{m g R n_{b} / 2}} 2^{(m g R-1) n_{b} / 2} \\
\sum_{i=0}^{2^{n_{b}-1}}\left(2 i-2^{n_{b} / 2}+1\right)=0
\end{array}
$$

since the mean value of the input symbols is null. Thus, the second derivative of the PGF in $z=1$ directly yields $\mathrm{E}\left[u_{n}^{2}\right]$ and $\mathrm{E}\left[v_{n}^{2}\right]$. 
The second derivative of (38) is

$$
\begin{aligned}
& G_{u}^{\prime \prime}(z)=G_{v}^{\prime \prime}(z)=\frac{m g R}{2^{m g R n_{b} / 2}}[(m g R-1) \\
& \left(\sum_{i=0}^{2^{n_{b} / 2}-1} z^{2 i-2^{n_{b} / 2}+1}\right)^{m g R-2} \\
& \sum_{i=0}^{2^{n_{b} / 2}-1}\left(2 i-2^{n_{b} / 2}+1\right) z^{2 i-2^{n_{b} / 2}}+ \\
& \left(\sum_{i=0}^{2^{n_{b} / 2}-1} z^{2 i-2^{n_{b} / 2}+1}\right)^{m g R-1} \sum_{\substack{i=0 \\
i \neq 2^{n_{b} / 2-1}}}^{2^{n_{b} / 2}-1}\left(2 i-2^{n_{b} / 2}\right. \\
& \left.+1)\left(2 i-2^{n_{b} / 2}\right) z^{2 i-2^{n_{b} / 2}-1}\right] \text {. }
\end{aligned}
$$

So,

$$
\begin{gathered}
\left.G_{u}^{\prime \prime}(z)\right|_{z=1}=\left.G_{v}^{\prime \prime}(z)\right|_{z=1}=\frac{m g R}{2^{m g R n_{b} / 2}} \\
\left\{(m g R-1) 2^{\frac{n_{b}}{2}(m g R-2)} \sum_{i=0}^{2^{n_{b}-1}}\left(2 i-2^{n_{b} / 2}+1\right)+\right. \\
2^{\frac{n_{b}}{2}(m g R-1)}\left[4 \sum_{i=0}^{2^{n_{b}-1}} i^{2}-\left(4 \cdot 2^{n_{b} / 2}-2\right) \sum_{i=0}^{2^{n_{b}-1}} i+\right. \\
\sum_{i=0}^{2^{n_{b}-1}}\left(2^{n_{b}}-2^{n_{b} / 2}\right)-\left(2 \cdot 2^{n_{b} / 2-1}-2^{n_{b} / 2}+1\right) \\
\left.\left.\left(2 \cdot 2^{n_{b} / 2-1}-2^{n_{b} / 2}\right)\right]\right\}=m g R \cdot 2^{-n_{b} / 2} \\
{\left[\begin{array}{c}
4\left(2^{n_{b} / 2}-1\right) 2^{n_{b} / 2}\left(2 \cdot 2^{n_{b} / 2}-2+1\right) \\
6 \\
\left(4 \cdot 2^{n_{b} / 2}-2\right) \frac{\left(2^{n_{b} / 2}-1\right) 2^{n_{b} / 2}}{2}+ \\
\left.2_{u}^{n_{b} / 2}\left(2^{n_{b}}-2^{n_{b} / 2}\right)\right] \Rightarrow \\
\left.G_{u}^{\prime \prime}(z)\right|_{z=1}=\left.G_{v}^{\prime \prime}(z)\right|_{z=1}=\frac{m g R}{3}\left(2^{n_{b}}-1\right) .
\end{array}\right.}
\end{gathered}
$$

As $u_{n}$ and $v_{n}$ are independent, the constellation average energy when $n_{b}>1$ is

$$
\begin{aligned}
E_{s} & =\mathrm{E}\left[\left|y_{n}\right|^{2}\right]=\mathrm{E}\left[u_{n}^{2}+v_{n}^{2}\right]=\mathrm{E}\left[u_{n}^{2}\right]+\mathrm{E}\left[v_{n}^{2}\right] \\
& =\frac{2 m g R}{3}\left(2^{n_{b}}-1\right) .
\end{aligned}
$$
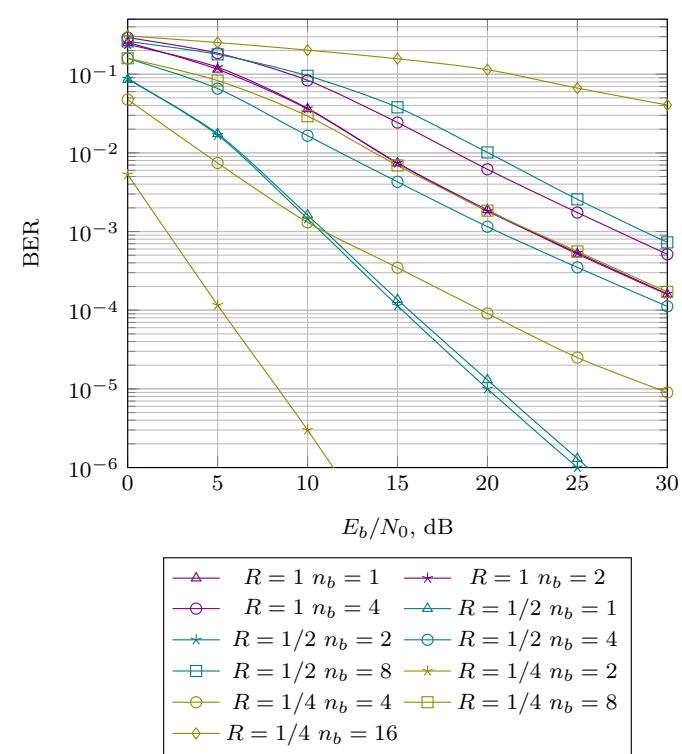

Fig. 7. Performances of the WCC system in Fig. 1 with a wavelet coding matrix $4 \times 16$ (rank $m=4$ and genus $g=4$ ) for a broad range of parameters. Curves for the same encoding rate have the same color and curves for the same value of $n_{b}$ employ the same point mark.

\section{PERFORMANCE ANALYSis}

In this Section, the performance of the proposed WCC system is evaluated by simulation. Firstly the consequences of variation of wavelet code parameters on the system performance is examined. Then, its performance is compared with the performance of ordinary symbol-by-symbol detecting systems and space-time block coding (STBC) systems.

\section{A. Performance of WCC systems}

In order to obtain a broad view of performance of the WCC system proposed in Fig. 1, it was simulated considering a wide range of parameters. Wavelet coding matrices with ranks $m=2$ and 4 , genera $g=4,16$ and 64 , coding rates $R=1,1 / 2$ and $1 / 4$, and data mapping with $n_{b}=1,2,4,8$ and 16 bits per input symbol were considered.

Fig. 7 presents performance curves of a WCC system employing a wavelet coding matrix $4 \times 16$ (rank $m=4$ and genus $g=4$ ) for some parameter variations. The cases were limited to spectral efficiencies (see Equation (25)) of 1, 2 and 4 bits/s/Hz. In Fig. 7, curves for the same encoding rate have the same color and curves for the same value of $n_{b}$ employ the same point mark (e.g., blue curves have $R=1 / 2$ and curves with $n_{b}=2$ employ $\star$ as point mark). 


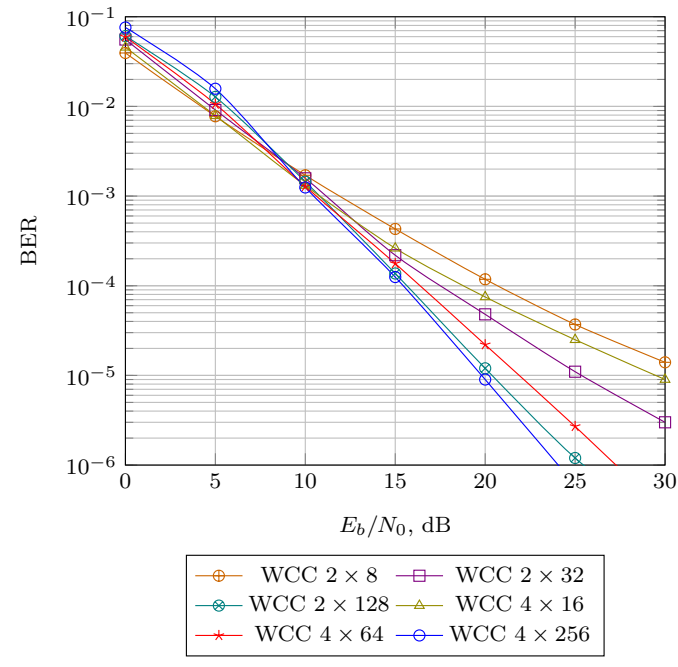

Fig. 8. Performances of the WCC system in Fig. 1 with encoding rate $R=1 / 4$ and data mapping with $n_{b}=4$ for wavelet coding matrices $2 \times 8,2 \times 32,2 \times 128,4 \times 16,4 \times 64$ and $4 \times 256$ (ranks $m=2$ and 4 and genus $g=4,16$ and 64 ).

Fig. 7, as expected, displays that decreasing rates result in better performance while increasing $n_{b}$, on the contrary, worsens that. Unfortunately, the first does not compensate the second for the same spectral efficiency and the employment of greater values of $n_{b}$ rapidly degrades system's performance. An exception occurs when $n_{b}$ goes from 1 to 2 , case in which similar performances are obtained for the same encoding rate (modulation with $n_{b}=2$ corresponds to modulations with $n_{b}=1$ in quadrature). Furthermore, for the same spectral efficiency, the best WCC system performance is obtained employing $n_{b}=2$. Similar behavior is obtained for any other wavelet coding matrix $m \times m g$.

Fig. 8 presents performance curves of WCC systems with encoding rate $R=1 / 4$ and data mapping with $n_{b}=4$ for wavelet coding matrices $2 \times 8,2 \times 32,2 \times 128,4 \times 16,4 \times 64$ and $4 \times 256$ (ranks $m=2$ and 4 and genera $g=4,16$ and 64).

We observe in Fig. 8 that increases in rank or genus (increasing the constraint length) of the wavelet matrix yield better performances of the proposed WCC system over a fading channel, mainly at higher signal-to-noise ratios. It happens because the correlation decoder employed here takes advantage of the input information spread over the transmitted symbols. A sequential decoding must take greater advantage of that and yield a better performance for a longer constraint length. This fact was verified in [16] with the employment of a Viterbi decoder. Future works shall verify it for other sequential decoders, as the Fano algorithm and the $M$-algorithm.

\section{B. Comparison with other systems}

In order to evaluate the WCC ability to improve system performance over a flat Rayleigh fading channel, the WCC system performance is compared with the performance of ordinary symbol-by-symbol detecting systems and STBC systems proposed in [21] considering two transmitting and one receiving antennas.

As WCC symbol-by-symbol decoding are employed here, it is more natural to compare its performance with ordinary symbol-by-symbol detecting systems. The symbol-by-symbol detecting systems simulated were BPSK for a spectral efficiency of $1 \mathrm{bit} / \mathrm{s} / \mathrm{Hz}$, QPSK for $2 \mathrm{bits} / \mathrm{s} / \mathrm{Hz}$ and 16-QAM for $4 \mathrm{bits} / \mathrm{s} / \mathrm{Hz}$.

STBC systems' performance is taken here as a reference of a powerful technique employed in modern systems. A performance comparison between WCC and STBC is not quite fair, since WCC is as simple as systems employing symbol-by-symbol decoding and only one transmitting and one receiving antennas, without spatial diversity as in STBC systems. Thus, it is not expected that WCC provides in general better performance than STBC. In fact, it is expected that a spatial diversity system may be developed from WCC, which can be explored in future works.

STBC was simulated employing the generating matrix

$$
\mathbf{G}=\left[\begin{array}{cc}
s_{1} & s_{2} \\
-s_{2}^{*} & s_{1}^{*}
\end{array}\right],
$$

where $s_{1}$ and $s_{2}$ are two transmitted signals considering as modulation BPSK for a spectral efficiency of $1 \mathrm{bit} / \mathrm{s} / \mathrm{Hz}, \mathrm{QPSK}$ for $2 \mathrm{bits} / \mathrm{s} / \mathrm{Hz}$ and 16-QAM for $4 \mathrm{bits} / \mathrm{s} / \mathrm{Hz}$.

Fig. 9 shows performance curves of WCC, BPSK and STBC systems for a spectral efficiency of $1 \mathrm{bit} / \mathrm{s} / \mathrm{Hz}$. WCC systems with wavelet matrices $2 \times 8$, $2 \times 32$ and $2 \times 128$, considering $R=1 / 2$ and $n_{b}=2$, exhibited better performance than BPSK and STBC systems. STBC presented performance better than WCC just for the smaller constraint length (wavelet matrix $2 \times 8$ ) for $E_{b} / N_{0}>23 \mathrm{~dB}$.

Fig. 10 presents performance curves of WCC, QPSK and STBC systems for a spectral efficiency of $2 \mathrm{bit} / \mathrm{s} / \mathrm{Hz}$. The WCC system presented better 


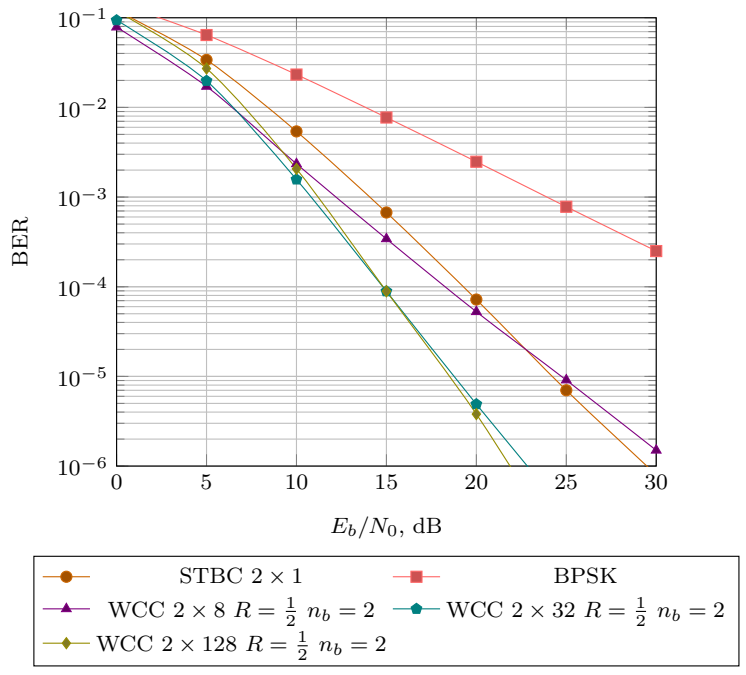

Fig. 9. Comparison between WCC, BPSK and STBC system performances with a spectral efficiency of $1 \mathrm{bit} / \mathrm{s} / \mathrm{Hz}$.

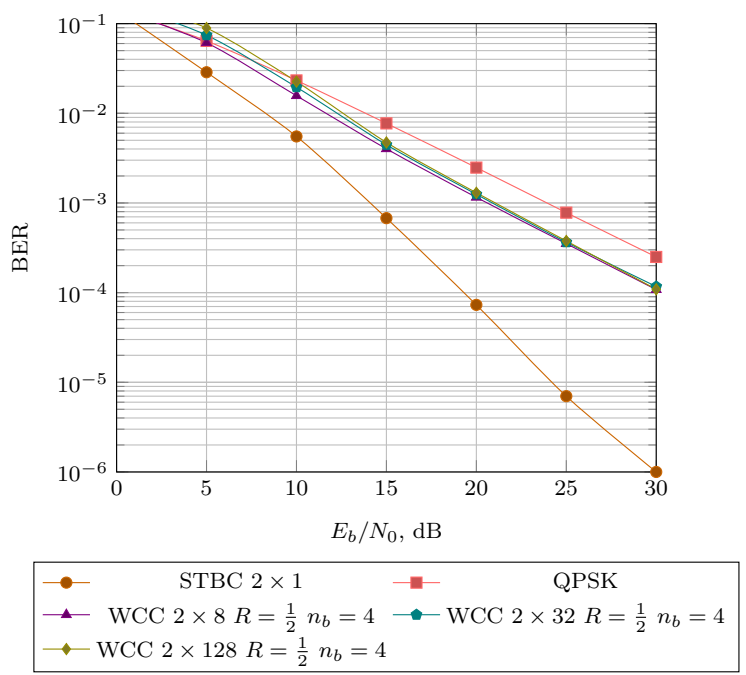

Fig. 10. Comparison between WCC, QPSK and STBC system performances with a spectral efficiency of $2 \mathrm{bit} / \mathrm{s} / \mathrm{Hz}$.

performance than QPSK. Differently than the former case, WCC performance was worse than STBC performance. The WCC performance was restrained by the rise of $n_{b}$ in order to attain greater spectral efficiency.

Fig. 11 presents performance curves of WCC, 16QAM and STBC systems for a spectral efficiency of $4 \mathrm{bit} / \mathrm{s} / \mathrm{Hz}$. A similar behavior may be seen in Fig. 10: higher values of $n_{b}$ degraded the WCC system performance. The WCC system presented worse performance than 16-QAM, except for the longer constraint length (wavelet matrix $2 \times 128$ ) in $E_{b} / N_{0}>19 \mathrm{~dB}$.

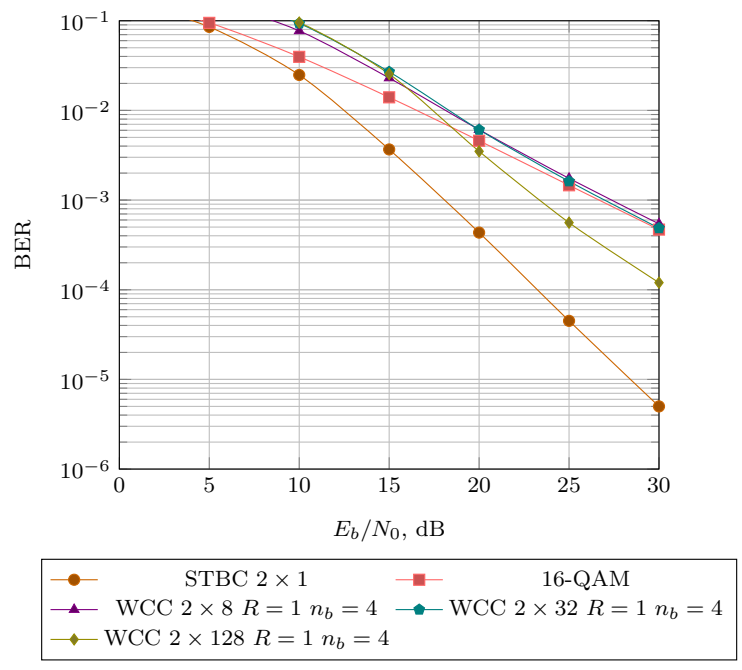

Fig. 11. Comparison between WCC, 16-QAM and STBC system performances with a spectral efficiency of $4 \mathrm{bit} / \mathrm{s} / \mathrm{Hz}$.

\section{CONCLUSIONS}

This paper presented a more general analysis of WCC considering flat real wavelet matrices derived from a Haar matrix and complex input symbols produced by a data mapper and representing $n_{b}$ source bits. WCC encoding and decoding processes were algebraically described and a probability distribution was formulated for the generated wavelet symbols. Also, signal constellations were proposed for transmission of these wavelet symbols and PGFs were calculated for them and used to deduce the average energy of the constellations.

The performance analysis of this system over a flat Rayleigh channel revealed that increasing rank or genus of the wavelet coding matrix yielded better performances of the proposed WCC system, specially for high signal-to-noise ratios. It happened because the correlation decoder employed here take advantage of the input information spread over the transmitted symbols. Future works should explore the employment of sequential decoders, as the Fano algorithm and the $M$-algorithm, instead of the correlation one. Sequential decoding for WCC may yield important performance increases, specially for large constraint lengths, at the expense of decoding complexity.

We also observed that the data mapping process employed prior to the WCC encoding degraded the system performance, whereas it allowed to increase the spectral efficiency. For a spectral efficiency of $1 \mathrm{bit} / \mathrm{s} / \mathrm{Hz}$, the WCC system presented a performance 
better than an ordinary symbol-by-symbol detecting system and even an STBC system with diversity 2 and the same spectral efficiency. For greater spectral efficiencies, when $n_{b}$ increased, the WCC system performance got worse. However, despite the observed degradation due to increases in $n_{b}$, we found great potential possibilities of application of WCC, since it showed better performances than ordinary symbol-by-symbol detecting systems, particularly at higher signal-to-noise ratios for higher spectral efficiencies.

Many critics pointed to the low spectral efficiency provided by WCC as its main disadvantage. Further improvements in this regard are planned for future works. Still as future work, as successive wavelet symbols in WCC are generated from a matrix with consistent orthogonality properties, one may investigate its employment as a diversity system. Application of WCC in multi-band ultra wideband systems (UWB) may also be investigated, since subbands of UWB may be coded with Hadamard codes and real flat wavelet matrices of genus $g=1$ are Hadamard matrices [24].

\section{REFERENCES}

[1] H. L. Resnikoff and R. O. Wells-Jr, Wavelet analysis: the scalable structure of information. New York: Springer-Verlag, 1998.

[2] M. A. Tzannes and M. C. Tzannes, "Bit-by-bit channel coding using wavelets," in Proc. IEEE Global Telecommunications Conference - GLOBECOM'92, pp. 684-688, 1992. doi: 10.1109/GLOCOM.1992.276430

[3] G. Forney, "Convolutional codes I: Algebraic structure," in IEEE Transactions on Information Theory, vol. 16, no. 6, pp. 720-738, Nov 1970. doi: 10.1109/TIT.1970.1054541

[4] L. F. Q. Silveira, Performance of systems with wavelet coding and space-time diversity over flat Rayleigh fading channels (in Portuguese). Master's Dissertation. Campina Grande/PB, Federal University of Campina Grande, 2002.

[5] L. F. Q. Silveira, F. M. de Assis, and E. L. Pinto, "Performance of a PSK system with wavelet time diversity over flat Rayleigh fading channels," in Proc. International Microwave and Optoelectronic Conference - IMOC, pp. 87-191, Jan., 2003. doi: 10.1109/IMOC.2003.1244855

[6] L. F. Q. Silveira, F. M. de Assis, and E. L. Pinto, "A new wavelet coded PSK system over time-varying flat fading channels," in Proc. Workshop on Signal Processing Advances in Wireless Communications - SPAWC, pp. 429-433, Jul., 2004. doi: 10.1109/SPAWC.2004.1439279

[7] L. F. Q. Silveira, Analysis of wavelet coding in systems subject to flat Rayleigh fading (in Portuguese). Doctoral Thesis. Federal University of Campina Grande. Campina Grande/PB, 2006.

[8] L. F. Q. Silveira, L. G. Q. Silveira Jr., F. M. de Assis, and E. L. Pinto, "Analysis and optimization of wavelet-coded communication systems." IEEE Transactions on Wireless Communications, Vol. 8, No. 2, pp. 563-567, Feb. 2009. doi: 10.1109/TWC.2009.070978
[9] L. G. Q. Silveira Jr., L. F. Q. Silveira, F. M. de Assis, and E. L. Pinto, "Soft-decision demodulation of wavelet-coded PSK signals over flat Rayleigh fading channels," in Proc. VI International Telecommunications Symposium - ITS, pp. 758-763, Sep., 2006. doi: 10.1109/ITS.2006.4433374

[10] L. G. Q. Silveira Jr., L. F. Q. Silveira, F. M. de Assis, and E. L. Pinto, "Interative decoding for serial concatenation with wavelet encoding," in Proc. International Microwave and Optoelectronics Conference - IMOC, pp. 743-747, Jul., 2007. doi: 10.1109/IMOC.2007.4404367

[11] L. G. Q. Silveira Jr., Iterative decoding in systems with wavelet coding (in Portuguese), Campina Grande, Doctoral Thesis, Federal University of Campina Grande, Feb. 2008. http://livros01.livrosgratis.com.br/cp067426.pdf

[12] E. A. Santos, E. C. Gurjão, and F. M. de Assis, "Design of signal constellations for wavelet coding (in Portuguese)," in Proc. XXIX Brazilian Communications Symposium - SBrT2011, Oct. 2011. doi: 10.14209/SBRT.2011.26

[13] E. A. Santos, E. C. Gurjao, and F. M. de Assis, "Increasing the spectral efficiency of systems with wavelet coding (in Portuguese)," in Proc. XXXI Brazilian Communications Symposium - SBrT2013, Sep. 2013. doi: 10.14209/SBRT.2013.31

[14] E. A. Santos, Transmission system with wavelet coding of arbitrary spectral efficiency (in Portuguese). Doctoral Thesis. Federal University of Campina Grande. Campina Grande/PB, 2014.

[15] J. Fonseca Neto, F. M. de Assis, E. C. Gurjão, and L. B. S. Lima, "Wavelet constellation algebraic construction (in Portuguese)," in Proc. XXXIII Brazilian Communications Symposium - SBrT, Juiz de Fora-MG, SBrT15, Sep. 2015. doi: 10.14209/SBRT.2015.101

[16] J. Fonseca Neto, L. B. S. Lima, and F. M. de Assis, "Viterbi decoding for channel wavelet coding (in Portuguese)," in Proc. XXXV Brazilian Communications and Signal Processing Symposium - SBrT, São Pedro/SP, SBrT17, Sep. 2017. doi: 10.14209/SBRT.2017.140

[17] L. C. Cavalcante, A. I. F. Rego, M. A. C. Fernandes and L. F. de Q. Silveira, "Performance analysis of wavelet coding in frequency selective channels (in Portuguese)," in Proc. XXXI Brazilian Communications Symposium - SBrT2013, Sep. 2013. doi: 10.14209/SBRT.2013.80

[18] L. G. Q. Silveira Jr., "Reduced-complexity iterative decoder for wavelet-coded systems in flat fading Channels," in Proc. 21st International Conference on Telecommunications - ICT, pp. 374 379, Jun. 2014. doi: 10.1109/ICT.2014.6845142

[19] L. G. Q. Silveira Jr. and L. F. Q. Silveira, "MMSE-based receiver for wavelet-filtered systems over flat fading channels," in Proc. 2016 European Conference on Networks and Communications EuCNC, pp. 1-7, Jun. 2016. doi: 10.1109/EuCNC.2016.7560994

[20] L. C. P. Cavalcante, S. Rommel, R. Diniz, L. G. Q. Silveira Jr., L. F. Q. Silveira, and I. T. Monroy, "Performance evaluation of wavelet-coded OFDM on a $4.9 \mathrm{~Gb} / \mathrm{s}$ w-band radio-over-fiber link," Journal of Lightwave Technology, Vol. 35, № 14, pp. 2803-2809, Jul. 2017. doi: 10.1109/JLT.2017.2701358

[21] S. M. Alamouti, "A simple transmitter diversity scheme for wireless communications," in Proc. IEEE Journal Selected Areas Communications, vol. 16, number 8, pp. 1451 - 1458, Oct. 1998. doi: $10.1109 / 49.730453$

[22] A. Papoulis and S. U. Pillai, Probability, Random Variables, and Stochastic Processes, 4. ed. McGraw-Hill Higher Education, 2002.

[23] J. G. Proakis, Digital communications, 5th ed., New York:McGraw-Hill, 2008.

[24] X. Gao, R. Yao, and Z. Feng, "Multi-band UWB system with Hadamard coding," in Proc. IEEE 58th Vehicular Technology Conference, Oct. 2003. doi: 10.1109/RWS.2007.351878 


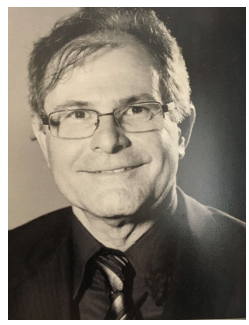

João Fonseca Neto received a Bachelor's Degree in Electrical Engineering (Electrical) from the Federal University of Paraiba (UFPB), Campina Grande, Paraíba, Brazil, from 1987. He also has a Master's Degree in Eletrical Engineering from the Federal University of Sergipe (UFS), Aracaju-SE, Brazil, in 2013, and a Doctor's Degree in Eletrical Engineering (Telecommunications) from the Federal University of Campina Grande (UFCG), Campina Grande-PB, Brazil, in 2018. From 1987 to 1991, he worked as researcher on microwaves at the Institute of Advanced Studies (IEAv) of the Aerospace Technical Center (CTA) (now called Brazilian Department of Science and Aerospace Technology) in São José dos Campos, São Paulo, Brazil. From 1999 to 2006, he worked as Manager of the National Telecommunications Agency in the state of Sergipe. From 2007 to 2013, he worked as technical engineer for Aperipê Foundation (Sergipe, Brazil), working on the transition from analogue to digital television, among others activities. He is currently professor at the Federal Institute of Sergipe. His field of study is microwave and telecomunications systems.

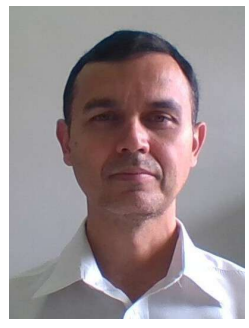

Leocarlos B. S. Lima is Electrical Engineer graduated in 1997 by Universidade Federal de Campina Grande (UFCG), PhD in Electrical Engineering since 2004 by UFCG and École Nationale Supérieure des Télécommunications (ENST). Currently, he is Professor and Administrative Coordinator of the Academic Unit of Electrical Engineering (UAEE) at UFCG. His main interests include channel coding.

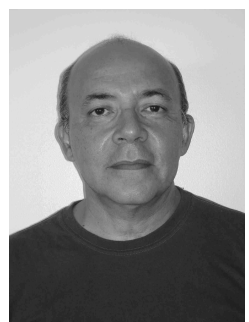

Francisco M. de Assis received the B.Sc. degree in 1984 and M.Sc. degree in 1992 in electrical engineering both from Military Institute of Engineering (IME), Rio de Janeiro, Brazil and, doctorade degree in 1994 from Pontifical Catholic University of Rio de Janeiro (PUC-Rio), Rio de Janeiro, Brazil. Currently he is Full Professor at Federal University of Campina Grande (UFCG) and head of the Institute for Studies in Quantum Computation and Quantum Information (IQuanta), Campina Grande, Paraíba, Brazil. His research interests include information theory applications to classical and quantum systems. 